\title{
Tuberculous cavernositis of the penis: case report
}

\author{
V RAMESH, * R VASANTHI $\dagger$ \\ From the departments of *Dermatology and $\uparrow$ Surgery, Safdarjung and Dr Ram Manohar Lohia Hospitals, New \\ Delhi, India
}

SUMMARY The case of an elderly man with tuberculous cavernositis of the penis leading to distortion and sinus tract formation is reported. The condition resembled carcinoma. Histopathology, a positive tuberculin test result, and a good response to treatment aided in making the diagnosis.

Tuberculosis of the penis is an extremely rare condition. Even before antibiotics had been discovered, when tuberculosis was prevalent, infection of the penis was recorded in only two out of 342 cases of tuberculosis of the genitourinary tract. ${ }^{1}$ The present report describes this uncommon condition, which can mislead the unwary clinician.

\section{Case report}

A man aged 78 presented with penile deformity and ulceration of nine months' duration. It had started as a papule on the glans penis, which increased in size and ulcerated. In the absence of specific treatment, the condition spread to the whole of the glans penis and penile shaft. The resultant thickening subsequently caused lateral deviation of the penis, and urine was passed through two or three orifices on the ventral surface of the glans penis near the urethral opening. The patient had no history suggestive of renal colic, haematuria, dysuria, or increased frequency of micturition. Two months previously he had elsewhere twice undergone a biopsy of the ulcer because carcinoma was suspected. The reports showed pseudoepitheliomatous hyperplasia and signs of chronic inflammation, but no signs of malignancy.

On examination, the prepuce was retracted and the glans penis was hard, shrunken, covered with a thick adherent crust at the lower end, and deviated to the right (figure). Removal of the crust showed an erosion with irregular margins. The distal half of the penile shaft was hard, nodular, and non-tender. The remaining areas of the penis and the scrotal contents were normal. The patient had no appreciable inguinal lymphadenopathy. Results of routine blood tests, urine analysis, and liver and renal function tests were

Address for reprints: Dr V Ramesh, Sector 1/696, RK Puram, New Delhi 110022, India

Accepted for publication 10 October 1988 within normal limits. The Venereal Disease Research Laboratory (VDRL) test gave a negative result. The Mantoux test (with one tuberculin unit of purified protein derivative) read $26 \times 20 \mathrm{~mm}$. Urine specimens sent on three separate occasions for culture for Mycobacterium tuberculosis gave negative results. Chest and abdomen radiography and excretory urography showed normal results. Biopsy of a nodule showed pronounced infiltration of the cavernous

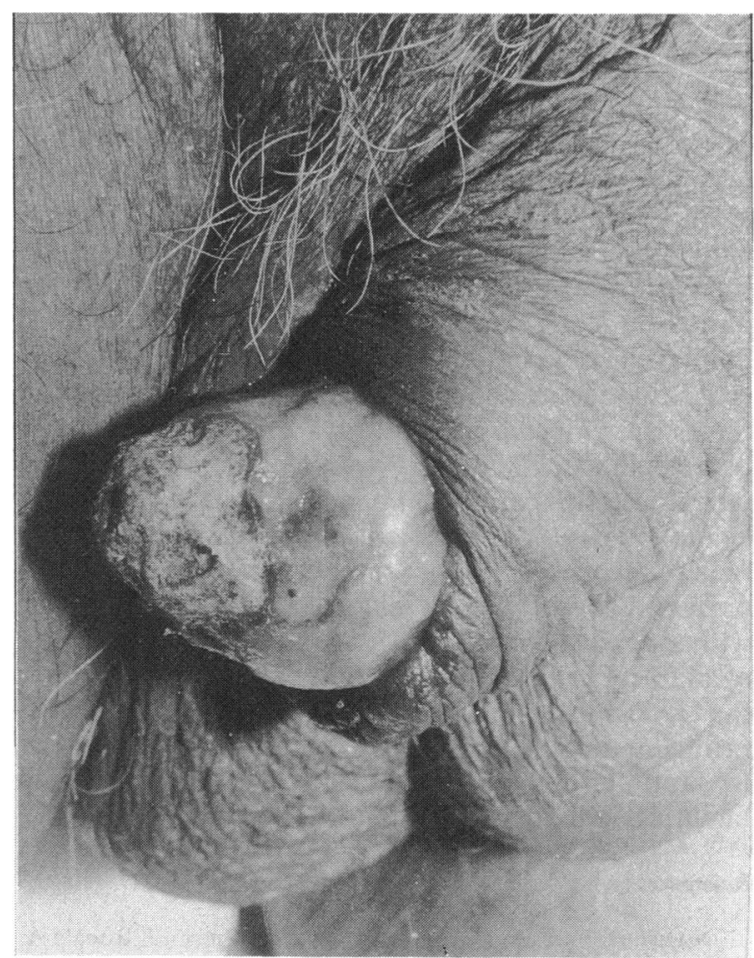

Figure Laterally deviated penile shaft with adherent crust on glans penis. 
tissue by lymphocytes, occasional neutrophils, histiocytes, and epithelioid cells and tubercle formation in some areas. A fibrous reaction was seen around the tubercles. Stains for acid fast bacilli showed no organisms.

Oral treatment with rifampicin $450 \mathrm{mg}$, isoniazid $300 \mathrm{mg}$, and thiactazone $150 \mathrm{mg}$ was given daily. Two months later the erosions of the glans penis showed signs of healing, and the progression of the disease was halted. During his latest visit six months after treatment the induration of the penile shaft had decreased considerably and the sinus tracts had almost healed.

\section{Discussion}

Tuberculosis of the penis usually presents as a primary lesion, though it may be secondary to an active focus at other sites in the body. Very rarely a severe necrotic form (called orificial tuberculosis) can occur in , debilitated people because of autoinoculation of the bacilli from progressive genitourinary tuberculosis. Papulonecrotic tuberculids of the glans penis, a relatively mild form of the disease, has been reported from Japan. ${ }^{2}$ The lesion can be an erythematous or keratotic plaque over the penile skin (called lupus vulgaris), urethritis, slowly progressive ulcer(s) on the glans penis, ${ }^{3-6}$ or (less commonly) cavernositis, ${ }^{3}$ which can scar and distort the penis, sometimes leading to sinus tract formation.

In our patient the lesion was of the primary type and started on the glans penis. Because of the delay in diagnosis, the tuberculous infiltration spread into the cavernous tissue, which led to nodularity, fibrosis, deformity, and the formation of sinus tracts. The hardness and apparent lack of pain suggested carcinoma. Histopathology and a positive tuberculin test result helped to make a diagnosis, which was confirmed by a good response to treatment. The previously poor prognosis for penile tuberculosis no longer applies since the discovery of effective treatment.

\section{References}

1 Lazarus JA, Arthur AR. Primary tuberculosis of the penis. J Urol 1936;35:361-77.

2 Nishigori C, Taniguchi S, Hayakawa M, Imamura S. Penis tuberculides: papulonecrotic tuberculides on the glans penis. Dermatologica 1986;172:93-7.

3 Lewis EL. Tuberculosis of the penis: a report of five cases and a complete review of the literature. J Urol 1946;56:737-45.

4 Lal MM, Sekhon GS, Dhall JC. Tuberculosis of the penis. J Indian Med Assoc 1971;56:316-8.

5 Agarwalla B, Mohanty GP, Sahu LK, Rath RC. Tuberculosis of the penis: report of two cases. $J$ Urol 1980;124:927.

6 Venkataramaiah NR, van Raalte JA, Dutta SN. Tuberculous ulcer of the penis. Postgrad Med J 1982;58:59-60. 